\title{
Signal to Noise Ratio of APD-Based Monolithic Scintillator Detectors for High Resolution PET
}

\author{
Marnix C. Maas, Dennis R. Schaart, D. J. (Jan) van der Laan, Herman T. van Dam, Jan Huizenga, J. C. Brouwer, \\ Peter Bruyndonckx, Cedric Lemaître, and Carel W. E. van Eijk, Member, IEEE
}

\begin{abstract}
Monolithic scintillator detectors, consisting of several $\mathrm{cm}^{3}$ of scintillating material coupled to one or more Hamamatsu S8550 avalanche photodiode (APD) arrays, are proposed as detectors for high resolution positron emission tomography (PET). In this work, the factors contributing to the variance on the signals are investigated, and their effects on the energy, time and spatial resolutions are analyzed.

Good agreement was found between a model of the energy resolution and experiments with a $20 \times 10 \times 10 \mathrm{~mm}^{3}$ LYSO:Ce crystal coupled to a single channel large-area APD (LAAPD). With the same crystal coupled to an APD array, differences between model and experiment were observed at high APD gain.

The measured energy resolution of $\sim 11 \%$ FWHM was dominated by scintillation photon statistics, with less important roles for the APD excess noise factor and electronic noise. On the other hand, electronic noise was an important factor both for the time and the spatial resolutions. The time resolution was found to depend strongly on the APD bias voltage, and was best at the highest bias. A time resolution of $1.6 \mathrm{~ns}$ full width at half maximum (FWHM) was measured against a $\mathrm{BaF}_{2}-\mathrm{PMT}$ detector. The best spatial resolution measured was $1.64 \mathrm{~mm}$ FWHM, without correction for the $\sim 0.9 \mathrm{~mm}$ FWHM measurement beam. It is estimated that an intrinsic spatial resolution of $1.26 \mathrm{~mm}$ FWHM can be achieved at the center of the detector with an infinitely narrow test beam.
\end{abstract}

Index Terms-Avalanche photodiode (APD), monolithic scintillator detector, positron emission tomography (PET), signal to noise ratio (SNR).

\section{INTRODUCTION}

$\mathbf{P}$ OSITRON EMISSION TOMOGRAPHY (PET) has gained a lot of interest in recent years as a molecular imaging tool for research on small animals such as rats and mice. The small dimensions of the organs of these animals impose stringent requirements on the spatial resolution and sensitivity of dedicated PET systems. Many current detector designs rely on small scintillation crystals to obtain the best possible spatial resolution [1]-[11]. However, the scanner sensitivity is reduced in such designs due to the dead space between the individual crystals. Additionally, the spatial resolution may be deteriorated by inter-crystal scatter and parallax errors due to depth of interaction (DOI) effects.

Manuscript received October 28, 2007; revised January 17, 2008.

M. C. Maas, D. R. Schaart, D. J. van der Laan, H. T. van Dam, J. Huizenga, J. C. Brouwer, and C. W. E. van Eijk are with the Delft University of Technology, 2629 JB Delft, The Netherlands (e-mail: m.c.maas@tudelft.nl).

P. Bruyndonckx and C. Lemaitre are with the Vrije Universiteit Brussel, 1050 Brussels, Belgium.

Digital Object Identifier 10.1109/TNS.2008.921493

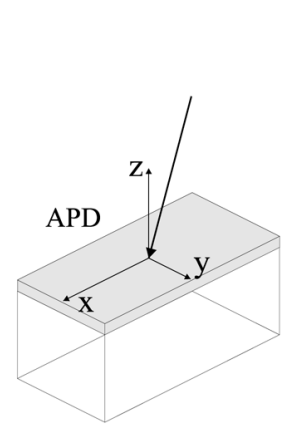

(a)

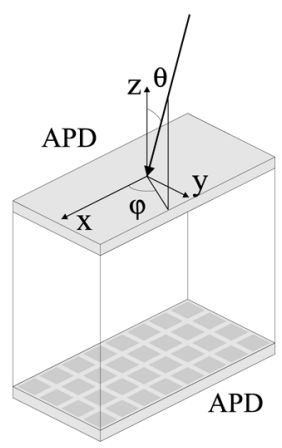

(b)
Fig. 1. Schematic representation of the detectors investigated. On the $10-\mathrm{mm}$ thick crystals (a), the APD array was coupled to the side facing the beam. On the 20 -mm thick crystals (b), the arrays were coupled to opposing sides.

To avoid these problems, monolithic scintillator detectors were proposed [12], [13]. These detectors consist of several $\mathrm{cm}^{3}$ of scintillating material coupled to one or more avalanche photodiode (APD) arrays (see Fig. 1). The entry point of an incoming annihilation photon on the front surface of the detector can be derived from the distribution of the scintillation light on the APD arrays. Because there are no individual crystal segments, sensitivity loss due to reflective material between the crystals is avoided.

Preliminary research on these detectors yielded promising results. A detector spatial resolution of $\sim 2.0 \mathrm{~mm}$ FWHM, not corrected for the $\sim 1 \mathrm{~mm}$ FWHM measurement beam was achieved with a 20-mm thick detector, with only a slight deterioration for angles of incidence up to $30^{\circ}$ [14]. This indicates that a thick layer of scintillating material can be used to maximize sensitivity, without deterioration of the image spatial resolution by parallax errors.

The aim of the present work is to gain a better understanding of the influence of signal variances on the detector energy, time and spatial resolutions, and of the factors that contribute to these signal variances. As the energy resolution represents a direct measure of a detector's signal-to-noise ratio (SNR), a model of this quantity for scintillator-APD detectors is presented. This model is first verified experimentally using a single channel large-area APD (LAAPD), in order to avoid effects such as differences in gain or excess noise factor between the individual channels of the APD arrays. It is subsequently also applied to the APD array detectors. Furthermore, analyses of the electronic noise and the time resolution of the APD array detectors are presented, and the influence of the signal variance on the spatial resolution is investigated. 


\section{THEORY}

\section{A. Energy Resolution}

For a scintillator coupled to an APD, the mean number of electrons $\bar{N}_{e}$ produced at the APD output due to incident radiation producing an average of $\bar{N}_{p h}$ scintillation photons per event can be written as

$$
\bar{N}_{e}=\eta \cdot \bar{M} \cdot \bar{N}_{p h}
$$

where $M$ is the APD multiplication gain, and $\eta$ represents the number of primary e-h pairs generated in the APD per scintillation photon, averaged over all photons per scintillation event and over all events. In this formula, $\bar{N}_{p h}$ and $\bar{N}_{e}$ represent averages over many events, while $\bar{M}$ is averaged over all primary electron-hole $(\mathrm{e}-\mathrm{h})$ pairs created in the APD conversion layer.

The energy resolution of this system, defined as the full width at half maximum (FWHM) of the full-energy peak divided by its centroid location, can be described by four terms. The first of these represents the variance in the number of photons generated per scintillation event, the second represents the added variance due to the conversion of scintillation photons to primary $\mathrm{e}-\mathrm{h}$ pairs in the APD, the third represents the APD multiplication variance, and the fourth represents the contribution of electronic noise [15]:

$$
\begin{aligned}
\frac{\Delta E}{E}=2.35\left(\frac{\operatorname{var}\left\{N_{p h}\right\}}{\bar{N}_{p h}^{2}}+\frac{1-\eta}{\eta \bar{N}_{p h}}\right. & \\
& \left.+\frac{1}{\eta \bar{N}_{p h}} \frac{\operatorname{var}\{M\}}{\bar{M}^{2}}+\frac{\mathrm{ENC}^{2}}{\left(\eta \overline{M N}_{p h}\right)^{2}}\right)^{1 / 2},
\end{aligned}
$$

where $\Delta E / E$ is the FWHM energy resolution, and ENC is the equivalent noise charge of the detector-amplifier system referred to the preamplifier input.

Due to e.g., non-proportionality of the scintillator electron response, the variance of $N_{p h}$ is often greater than what would be expected based on Poisson statistics. In this work, this broadening is represented by an excess variance factor $\alpha^{2}$ describing the observed photon variance relative to the Poisson variance:

$$
\alpha^{2}=\frac{\operatorname{var}\left\{N_{p h}\right\}}{\bar{N}_{p h}} .
$$

Assuming that this term is independent of the energy of the incident radiation in the range of energies considered in this work (511-662 keV), the same value of $\alpha$ can be used for model calculations at different energies. This assumption may be invalid at lower energies. This approach is also followed in the Monte Carlo code Geant4 [16] used in our simulations of monolithic scintillator detectors [17], and is included in this experimental model for ease of comparison.

The variance associated with the multiplication process in the APD is commonly expressed in terms of the excess noise factor $J$, which is defined as:

$$
J \equiv \frac{\operatorname{var}\{M\}}{\bar{M}^{2}}+1 .
$$

Using (2)-(4), the energy resolution may now be written as:

$$
\frac{\Delta E}{E}=2.35\left(\frac{\alpha^{2}-1}{\bar{N}_{p h}}+\frac{J}{\bar{N}_{e} / \bar{M}}+\frac{E N C^{2}}{\bar{N}_{e}^{2}}\right)^{1 / 2} .
$$

The first term in this equation represents the deviation from Poisson statistics of the number of scintillation photons produced per event. The Poisson statistics are contained in the second term, where the factor $\bar{N}_{e} / \bar{M}=\eta \bar{N}_{p h}$ represents the number of primary $\mathrm{e}-\mathrm{h}$ pairs.

\section{B. Equivalent Noise Charge}

Assuming equal shaping time constants for differentiation and integration, the squared equivalent noise charge of a radiation detector-amplifier system can be written as [18]:

$$
\begin{aligned}
& E N C^{2}=\frac{1}{q^{2}}\left(\frac{a C_{\mathrm{tot}}^{2} A_{1}}{\tau}\right. \\
& \left.\quad+\left[2 \pi a_{f} C_{\mathrm{tot}}^{2}+\frac{b_{f}}{2 \pi}\right] A_{2}+b A_{3} \tau\right),
\end{aligned}
$$

where $a$ is the spectral density of the series white noise, $b$ is the spectral density of the parallel white noise, $a_{f}$ is the coefficient of the series $1 / \mathrm{f}$ noise, and $b_{f}$ is the coefficient of parallel $1 / \mathrm{f}$ noise. $C_{\text {tot }}$ is the total input capacitance of the detector-preamplifier system, $q$ is the elementary electron charge, $\tau$ is the amplifier shaping time, and $A_{1}-A_{3}$ are dimensionless constants depending on the type of shaping network. A mathematical frequency range is assumed in this model: $-\infty<f<+\infty$.

The series white noise contribution is mainly due to thermal noise of the preamplifier input FET channel and may be written as [19]:

$$
a=\Gamma \frac{2 k_{b} T}{g_{m}},
$$

where $g_{m}$ is the FET transconductance, $k_{b}$ is Boltzmann's constant, $T$ is the temperature and $\Gamma$ is the FET channel form factor, having values ranging between 0.5 and 0.7 .

The parallel white noise contribution $b$ consists mainly of shot noise related to leakage currents through the detector and the preamplifier FET gate, and of thermal noise of the preamplifier feedback resistor. For a detector with internal amplification such as an APD, a distinction can be made between unamplified (surface) leakage current $I_{l s}$ and amplified (bulk) leakage current $I_{l b}$, resulting in a total detector leakage current of [20]:

$$
I_{l}=I_{l s}+\bar{M} I_{l b}
$$

Taking into account that the amplified portion of the leakage current also experiences the APD excess noise factor, the total white parallel noise contribution may be written as:

$$
b=q\left(I_{l g}+I_{l s}+J \bar{M}^{2} I_{l b}\right)+\frac{2 k_{b} T}{R_{f}} .
$$

where $I_{l g}$ is the FET gate leakage current and $R_{f}$ is the preamplifier feedback resistance. 


\section{Time Resolution}

The time resolution of a pair of detectors can be determined by measuring the distribution of time differences between prompt coincident events. In this work, the time resolution of the LYSO-APD array detectors is measured against a fast PMT detector, so that the variance of this distribution can be written as:

$$
\sigma_{t}^{2}=\sigma_{A}^{2}+\sigma_{P}^{2}
$$

where $\sigma_{A}^{2}$ and $\sigma_{P}^{2}$ are the variances introduced by the APD array detector and the PMT detector, respectively. The variance in the time pickoff of each of these detectors consists of contributions related with time variations in the interaction of the radiation with the detectors, variations in the amplitude and shape of the scintillation pulse, time variations induced by its detection and the associated electronics, and the time pickoff method [21]. The first of these contributions is neglected in this analysis in view of the small dimensions of the detectors.

When a constant fraction discriminator (CFD) is used for time-pickoff, the uncertainty of the zero-crossing time of its output bipolar pulse can be related to the signal fluctuations and the signal slope at the zero-crossing point, according to [22]:

$$
\sigma_{t_{0}}=\left(\frac{\sigma_{v}}{\mathrm{~d} v / \mathrm{d} t}\right)_{t=t_{0}}
$$

where $\sigma_{v}$ denotes the rms signal fluctuation, $\mathrm{d} v / \mathrm{d} t$ denotes the signal slope and $t_{0}$ represents the zero-crossing time. In the LYSO-APD array detector, the signal fluctuations described by $\sigma_{v}$ may be caused by statistical fluctuations in the pulse amplitude and shape, and by electronic noise. It is assumed that pulse amplitude variations are cancelled by the CFD. Additionally, the large number of primary $\mathrm{e}-\mathrm{h}$ pairs that are multiplied in the APD and integrated on the preamplifier feedback capacitance in each scintillation pulse, combined with the short scintillation decay time of LYSO ( $\sim 40 \mathrm{~ns})$ and the limited bandwidth of the preamplifier-fast amplifier system, result in a negligible contribution of pulse shape walk. Hence, it is assumed that only electronic noise contributes to $\sigma_{v}$.

The signal slope at the zero-crossing point is assumed to be proportional to the ratio of the pulse amplitude and rise time. The pulse amplitude is given by the pulse height in response to a single input electron, $V_{p k}^{\prime}$, times $N_{e}$. In the ratio $\sigma_{v} / V_{p k}^{\prime}$, the definition of equivalent noise charge is recognized. Hence, it follows that

$$
\sigma_{A} \sim \frac{E N C_{f}}{\bar{N}_{e} / t_{r}}
$$

where $t_{r}$ denotes the pulse rise time and the $f$ subscript is used to indicate the fast amplifier branch. Hence, to optimize the time resolution, the product of $E N C_{f}$ and $t_{r}$ should be minimized.

\section{Spatial Resolution}

In addition to scattering of the incident radiation inside the crystals, the SNR of the detector signals forms one of the main contributions to the detector spatial resolution [25]. To investigate the probability distribution of the positioning error due to statistics and electronic noise for a given, arbitrary event, it is assumed that (1) the covariance between the detector channels may be neglected, and (2) the probability distribution of the positioning error depends on the SNR of the signals measured for that given event only. A detailed statistical analysis of the spatial resolution obtainable with these detectors, which also takes the covariance between the channels into account, is presented elsewhere [23]. It is shown there that assumption (1) is indeed valid for the detectors investigated here. Assumption (2) can be justified by considering that the set of training data used by the positioning algorithm employed is large (see Section III.C), rendering the effects of statistics and noise in this latter set negligible [24], [25].

Summarizing, the probability distribution of the positioning error due to statistics and electronic noise of an event with (a) given energy deposition(s) at given location(s) in the crystal is assumed to depend only on the SNR per channel of that same event. For this given event, the photon detection efficiency $\eta_{i}$ of a channel $i$ is defined as the expectation value of the number of $\mathrm{e}-\mathrm{h}$ pairs generated in APD pixel $i$ divided by the expectation value of the number of emitted scintillation photons that corresponds to the energy deposited in the event. If this energy corresponds to an expectation value of the number of emitted scintillation photons equal to $\bar{N}_{p h}$, the relative signal variance in channel $i$ can be derived using (2), substituting $\eta_{i}$ for $\eta$ :

$$
\begin{aligned}
\left(\frac{\sigma_{i}}{\mu_{i}}\right)^{2}=\frac{\operatorname{var}\left\{N_{p h}\right\}}{\bar{N}_{p h}^{2}} & +\frac{1-\eta_{i}}{\eta_{i} \bar{N}_{p h}} \\
& +\frac{1}{\eta_{i} \bar{N}_{p h}} \frac{\operatorname{var}\{M\}}{\bar{M}^{2}}+\frac{E N C_{i}^{2}}{\left(\eta_{i} \overline{M N}_{p h}\right)^{2}},
\end{aligned}
$$

where $\mu_{i}, \sigma_{i}$ and $E N C_{i}$ represent the signal expectation value and standard deviation and the equivalent noise charge of channel $i$, respectively.

To assess the relative importance of each of the terms in (13), a hypothetical event is considered with the same total energy deposited, for which $\eta_{i}=\eta / n$, where $n$ is the number of detector channels. Thus, the light distribution of this event is expected to be uniform over the detector pixels. The independence of the electronic noise in the detector channels results in $E N C_{i}=E N C / \sqrt{n}$. This leads to the following expression:

$$
\left(\frac{\sigma_{i}}{\mu_{i}}\right)^{2}=\frac{\alpha^{2}-1}{\bar{N}_{p h}}+n \frac{J}{\bar{N}_{e} / \bar{M}}+n \frac{E N C^{2}}{\bar{N}_{e}^{2}}
$$

Thus, for this hypothetical situation, the relative importance of the terms representing excess noise and electronic noise is increased by a factor $n$ compared to (5), while the term corresponding to the excess scintillation photon variance remains unaltered. It is assumed that the relative influence of the various terms on the spatial resolution indicated by (14) is representative for other, arbitrary, events.

\section{MATERIALS}

\section{A. Crystals}

The crystals investigated in this work are listed in Table I. The sample names consist of the crystal material followed by 
TABLE I

LIST OF CRYSTALS USED IN THE EXPERIMENTS

\begin{tabular}{cccc}
\hline Sample & Material & $\begin{array}{c}\text { Dimensions } \\
\left(\mathrm{mm}^{3}\right)\end{array}$ & Vendor \\
\hline LYSO10-P1 & LYSO(Ce) & $20 \times 10 \times 10$ & Photonic Materials \\
LYSO10-P2 & LYSO(Ce) & $20 \times 10 \times 10$ & CrystalPhotonics \\
LYSO20-P1 & LYSO(Ce) & $20 \times 10 \times 20$ & Photonic Materials \\
LYSO20-P2 & LYSO(Ce) & $20 \times 10 \times 20$ & CrystalPhotonics \\
\hline
\end{tabular}

TABLE II

CHARACTERISTICS OF THE LAAPD AND THE APD ARRAYS

\begin{tabular}{lll}
\hline Device & LAAPD & APD arrays \\
\hline Manufacturer & Advanced Photonics & Hamamatsu \\
Type & $630-70-73-510$ & S8550 SPL \\
Sensitive area & $\varnothing 16 \mathrm{~mm}$ circular & $32 \times 1.6 \mathrm{~mm} \times 1.6 \mathrm{~mm}$ \\
Gain $^{\mathrm{a}}$ & 200 at $1850 \mathrm{~V}$ & $50 \mathrm{at} 400 \mathrm{~V}$ \\
Dark current $^{\mathrm{a}}$ & $280 \mathrm{nA}$ at Gain 200 & $10 \mathrm{nA} / \mathrm{ch}$ at Gain 50 \\
Capacitance $^{\mathrm{a}}$ & $130 \mathrm{pF}$ & $10 \mathrm{pF}$ per channel \\
\hline
\end{tabular}

${ }^{\text {a }}$ Typical values

the thickness in $\mathrm{mm}$, a letter representing the surface finish and a sample number. The crystals used in this work were all polished, represented by the letter P. The photon statistics contribution $\left(\alpha^{2}-1\right) / \bar{N}_{p h}$ of these crystals was measured on a PMT (Hamamatsu R1791), using an Ortec 672 shaping amplifier and an Ortec AD114 ADC.

\section{B. $\angle A A P D$}

LAAPD measurements were performed using an Advanced Photonix LAAPD model 630-70-73-510, serial no. 128-10-11 [26]. It was contained in a temperature controlled box, and was read out using a Goyot preamplifier [27], an Ortec 672 shaping amplifier and an Ortec AD114 ADC. Energy resolution measurements were performed with crystal LYSO10-P2, packed in Teflon powder, with a $10 \times 10 \mathrm{~mm}^{2}$ face optically coupled to the $\oslash 16 \mathrm{~mm}$ LAAPD.

\section{APD Arrays}

The APD arrays used in this work are Hamamatsu model S8550-SPL, serial numbers 036 and 037, see Table II. These arrays consist of 2 banks of $2 \times 8$ pixels measuring

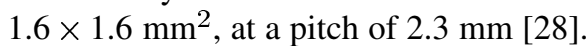

Each bank has a specific bias voltage $V_{50}$ at which the mean channel gain $\langle M\rangle=50$ according to manufacturer specifications. Between banks in the same array, and between different arrays, differences in $V_{50}$ of $10-20 \mathrm{~V}$ are common. In this work, the bias voltage of the arrays is therefore expressed in terms of $\Delta V \equiv V-V_{50}$, where $V$ is the applied bias voltage. The average gains $\langle M\rangle$ of the individual banks of the APD arrays used in this work, measured as a function of $\Delta V$ using $5.89 \mathrm{keV}$ $\mathrm{X}$-rays from an ${ }^{55} \mathrm{Fe}$-source, were found to coincide within $4 \%$ between $\Delta V=-40 \mathrm{~V}$ and $\Delta V=+10 \mathrm{~V}$. Breakdown effects started to occur at approximately $\Delta V=18 \mathrm{~V}$.

The experimental setup used to investigate the LYSO-APD array detectors is depicted in Fig. 2. The detectors are contained in a light-tight $\mathrm{Al}$ box, which is held at a constant temperature using Peltier coolers. To define a narrow beam of $511 \mathrm{keV}$ photons, a $\varnothing 0.5 \mathrm{~mm}{ }^{22} \mathrm{Na}$-source is used, and a second detector, consisting of a $19 \times 35 \mathrm{~mm}^{3} \mathrm{BGO}$ crystal coupled to a

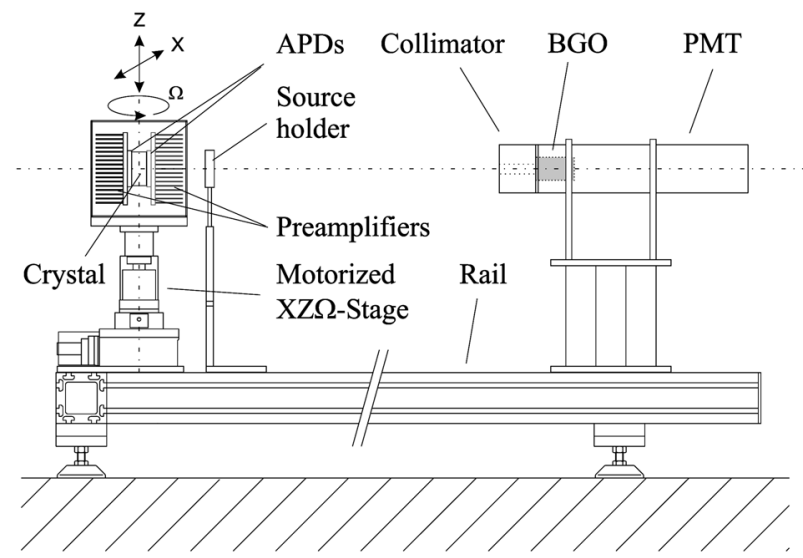

Fig. 2. Schematic representation of the experimental setup used to analyze the APD array detectors.

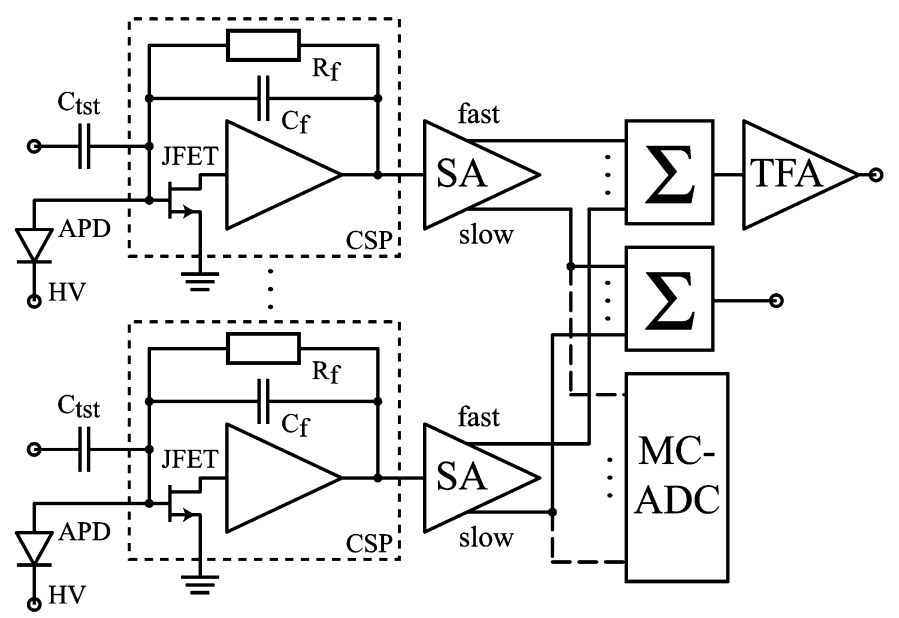

Fig. 3. Schematic representation of the front-end electronics. CSP: charge-sensitive preamplifier; SA: shaping amplifier; TFA: timing filter amplifier; MC-ADC: multichannel ADC.

PMT (XP2020) equipped with a Pb collimator, is used in coincidence with the APD detector. The width of the photon beam can be controlled by varying the distance between the PMT and the source. The 32 or 64 APD signals are each individually preamplified by Cremat CR-110 charge-sensitive preamplifiers (CSPs). Further amplification and shaping is provided by several CAEN N568BB 16-channel shaping amplifiers, which have a fast and a slow output branch. The slow output branch has a CR-(RC) ${ }^{2}$ shaping network with an adjustable gain and shaping times of $0.1,0.2,1$ and $3 \mu \mathrm{s}$; the fast output branch is a fixed-gain single differentiation stage with a time constant of $100 \mathrm{~ns}$. A schematic representation of the front-end electronics is shown in Fig. 3.

Measurements of the equivalent noise charge and the energy resolution were performed on the analog sum signal of the slow amplifier outputs. Pulse height spectra were obtained using an Ortec AD413A ADC.

Time resolution measurements were performed against a $25.4 \times 25.4 \mathrm{~mm}^{3} \mathrm{BaF}_{2}$ crystal coupled to an XP2020Q PMT. The PMT signal was directly fed into a constant fraction discriminator (CFD, Ortec 934) for time pickoff. Time pickoff on the APD detectors was performed by feeding the analog 
sum of the fast outputs of the linear amplifiers to a CFD, via a timing filter amplifier (TFA, Ortec 454) with a variable integration time constant. The time spectra were measured using a time-to-amplitude converter (TAC, Ortec 476), and an Ortec AD413A ADC. The time axis was calibrated with an Ortec 462 time calibrator.

Positional information can be extracted from the distribution of scintillation light on the APD arrays in each event. To record these, multichannel ADCs (CAEN V785) were used to read out each slow-output channel separately.

\section{METHODS}

\section{A. Scintillation Photon Variance}

The scintillation photon statistics term $\left(\alpha^{2}-1\right) / \bar{N}_{p h}$ was calculated by subtracting the PMT multiplication variance, calculated from the single electron spectrum, from the measured energy resolution [29]. The electronic noise contribution was assumed to be negligible.

\section{B. LAAPD Energy Resolution}

Calculations of the energy resolution of crystal LYSO10-P2 were performed, using separately measured values for the individual contributions in (5). The results of these calculations were compared to measured energy resolutions. To measure the LAAPD gain, the peak positions of spectra recorded with a pulsed laser (Hamamatsu C5143, $\lambda=667 \mathrm{~nm}$ ) were compared to the peak position at $V=400 \mathrm{~V}$, where unity gain was assumed. The excess noise factor was determined by measuring the energy resolution of the laser pulser at a fixed gain as a function of the number of output electrons, by inserting optical filters between the light source and the LAAPD, thus effectively varying $\eta$. The energy resolution observed with the laser pulser can be written as (see (2) and (5)):

$$
\left(\frac{\sigma\left(N_{e}\right)}{\bar{N}_{e}}\right)^{2}=h_{1}+\frac{h_{2}}{\bar{N}_{e}}+\frac{h_{3}}{\bar{N}_{e}^{2}},
$$

where $h_{1}=\operatorname{var}\left\{N_{p h}\right\} / \bar{N}_{p h}^{2}-1 / \bar{N}_{p h}$ is the deviation from Poisson statistics of the distribution of the number of photons produced per laser pulse. This term remains constant during the experiment. The term $h_{2}=J \bar{M}$ describes the excess noise contribution, and $h_{3}=E N C^{2}$ represents the electronic noise. The excess noise factor can thus be obtained by least-squares fitting this model to the data. An example of this type of experiment, performed at $M \sim 95$, is presented in Fig. 4. It is noted that with this method, no model terms need to be neglected, and no assumptions about the underlying statistics of photons or electron-hole pairs are made.

\section{APD Arrays}

1) Equivalent Noise Charge: The electronic noise of the APD arrays was investigated by measuring the equivalent noise charge at various APD bias and amplifier shaping time settings. Measurements were performed by injecting charge into calibrated $\sim 1.8 \mathrm{pF}$ capacitors at each of the CSP test inputs using

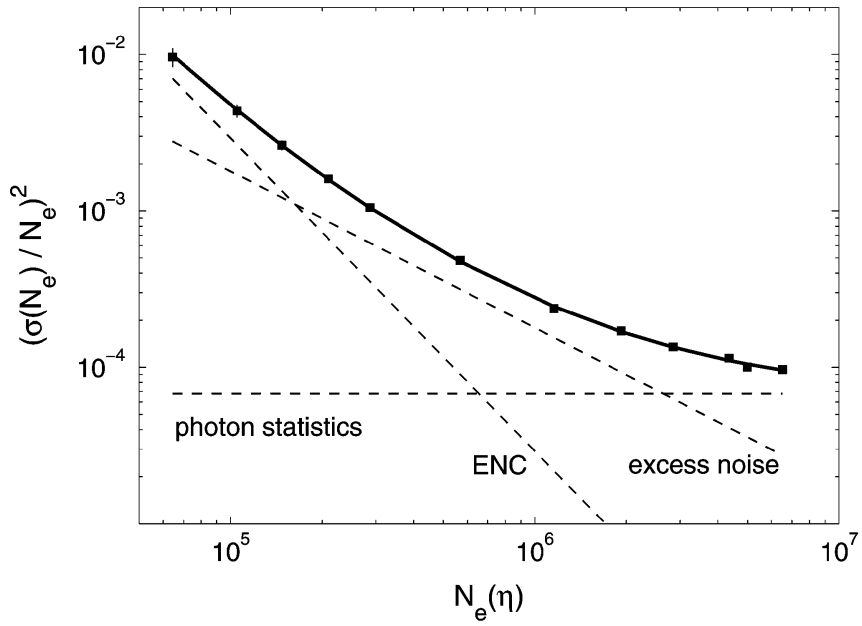

Fig. 4. Energy resolution of the laser pulser as a function of the number of output electrons, at a constant LAAPD gain of $\sim 95$. The solid line represents a least squares fit to the data using (15); the dashed lines indicate the individual components.

a square wave test pulser and obtaining pulse height spectra of the analog sum of the slow amplifier output signals.

2) Time Resolution: The influence of electronic noise and amplifier shaping on timing was investigated by measuring the time resolution at various TFA integration time settings. The influence of the APD bias voltage on the time resolution was also investigated.

3) Energy Resolution: Measurements of the energy resolution were performed at $511 \mathrm{keV}$ with the crystals listed in Table I. Calculations according to (5) were also done, again using separately measured values of the individual contributions.

Measurement of the gain of the APD arrays is not straightforward. Gain measurements using an optical source such as an LED or a pulsed laser are complicated by the fact that at low bias voltage, where unity gain is assumed, the pixels of the array are not fully depleted [20]. The possible change in the effective sensitive volume of the pixels with increasing bias voltage and the optical crosstalk caused by the $0.5 \mathrm{~mm}$ epoxy window in front of the pixels cause an uncertainty in the measured gain. These effects are illustrated by the significant differences reported between gain measurements with and without $\oslash 1.3 \mathrm{~mm}$ masks in front of the arrays [30].

To avoid these issues, the APD gain was measured with $5.89 \mathrm{keV} \mathrm{X}$-rays from a ${ }^{55} \mathrm{Fe}$-source in this work. X-ray measurements can however result in a significantly lower gain than measurements with optical photons. This effect depends on the X-ray energy, the APD gain and the device structure, [31], [32], and has indeed been observed for the $\mathrm{S} 8550$ arrays at $5.89 \mathrm{keV}$ [33].

The uncertainties in the gain measurements with an optical source preclude determination of the excess noise factor using the method described above. Determination of the ENF from $\mathrm{X}$-ray data is complicated by effects of non-uniformity of the gain over the detection area of each APD pixel. This effect can add significantly to the peak width observed with X-rays, but is averaged out when detecting optical photons which are spread 


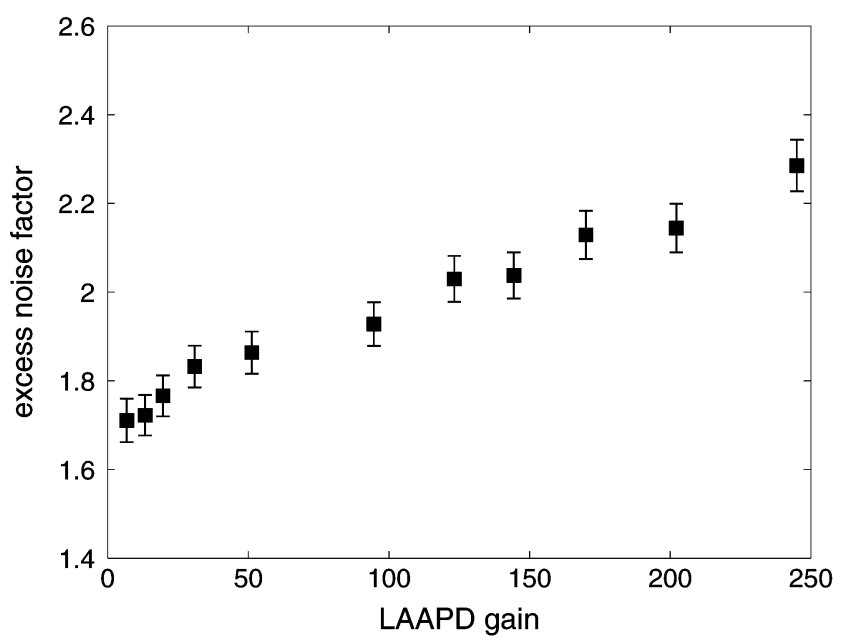

Fig. 5. Excess noise factor of the LAAPD as a function of the gain. The error bars indicate a $1 \sigma$ uncertainty.

over the whole detection area in each event [34]. An excess noise factor of $\sim 1.75$ has been reported elsewhere for these arrays, nearly independent of the APD gain [33]. This value was adopted in this work.

The model term $\bar{N}_{e} / \bar{M}$ describes the mean number of primary e-h pairs per scintillation event and is thus independent of $M$. The term was determined by comparing the peak position of the pulse height spectrum with that of a square-wave test pulser. This was done at a low gain $(M \sim 18)$, where the difference between optical and X-ray gain is assumed to be small.

4) Spatial Resolution: Spatial resolution measurements were performed by scanning the detectors through the beam in steps of $0.25 \mathrm{~mm}$ along the $x$ or the $y$ axis of the crystals at normal incidence (see Fig. 1). At each beam position, 1500 light distributions were recorded, consisting of 32 or 64 channel values from the multichannel ADCs. The coordinate of each event was estimated with a statistical learning algorithm based on the nearest neighbor method [35], using the rest of the same data set as training data (leave-one-out approach). With both the estimated and the real beam coordinates known, a histogram of the positioning errors can be created. The FWHM and FWTM of the overall error histogram containing all events in a data set served as a measure of the spatial resolution for the purposes of this work. These numbers were obtained by linear interpolation of the histograms. This position estimation algorithm and its performance optimization are described in more detail elsewhere [36]. In the present work, the position estimation algorithm was operated using 500 nearest neighbors.

\section{RESULTS AND DISCUSSION}

\section{A. Scintillation Photon Variance}

The photon statistics contribution $\left(\alpha^{2}-1\right) / \bar{N}_{p h}$ was measured both at $662 \mathrm{keV}$ and at $511 \mathrm{keV}$. For constant $\alpha$, the ratio of both photon statistics terms should equal the ratio of the photon yields, i.e., $662 / 511=1.295$. Experimentally, a ratio of 1.285 was observed, indicating that $\alpha$ may indeed be regarded constant in the energy range considered.

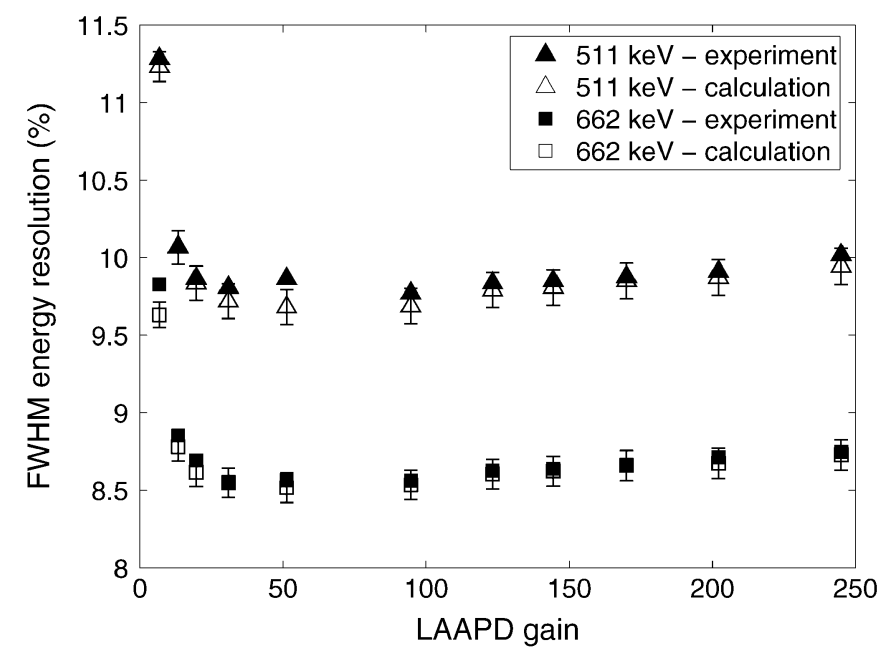

Fig. 6. Measured and calculated energy resolution of crystal LYSO10-P2 coupled to the LAAPD as a function of the gain, at $662 \mathrm{keV}$ and $511 \mathrm{keV}$. The error bars indicate a $1 \sigma$ uncertainty. The error bars of the experimental data fall within the markers.

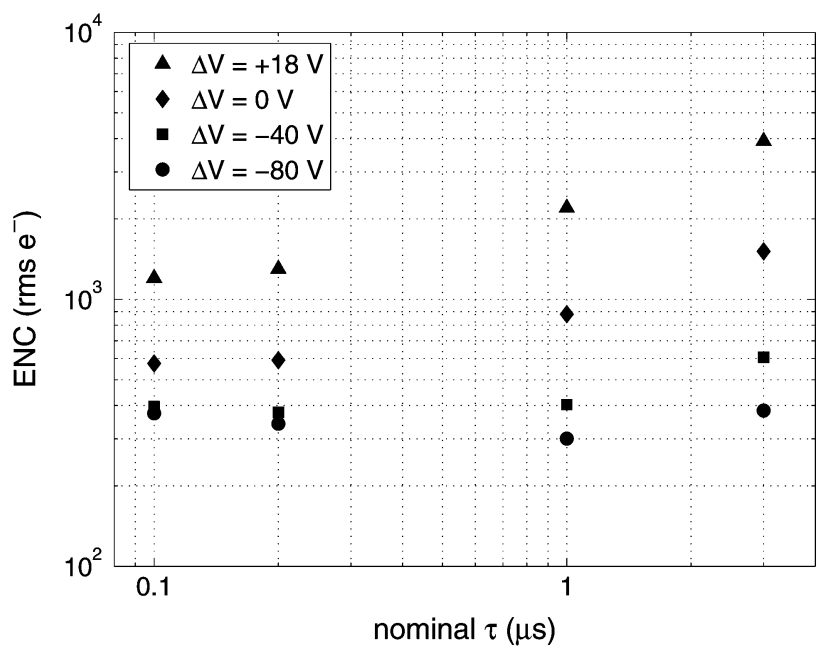

Fig. 7. ENC of a single detector channel as a function of the nominal amplifier shaping time $\tau$ at various APD bias voltages.

\section{B. LAAPD Energy Resolution}

The excess noise factor of the LAAPD is plotted as a function of the gain in Fig. 5. A comparison of the calculated and measured energy resolutions of crystal LYSO10-P2 at $662 \mathrm{keV}$ and $511 \mathrm{keV}$ as a function of the LAAPD gain is presented in Fig. 6. Good agreement between calculation and experiment is observed at both energies.

\section{APD Arrays}

1) Equivalent Noise Charge: Fig. 7 shows measurements of the equivalent noise charge of a single detector channel as a function of the nominal amplifier shaping time, at several APD bias voltages. At $\Delta V=0 \mathrm{~V}$, the best ENC is found at $\tau=$ $0.1 \mu$ s with a value of $\sim 550 \mathrm{e}^{-} \mathrm{rms}$. The same measurement performed on the analog sum signal of 64 channels yielded an average of $\sim 600 \mathrm{e}^{-}$per channel.

It is customary to fit the data of Fig. 7 to the model in (6) in order to estimate the individual noise contributions. However, it 
TABLE III

TIME RESOLUTION OF LYSO20-P2 AS A FUNCTION OF $\tau_{i}$ AT DIFFERENT BIAS VOLTAGES

\begin{tabular}{cccc}
\hline \multirow{2}{*}{$\tau_{i}$} & $t_{r}(10 \%-90 \%)$ & \multicolumn{2}{c}{ Time resolution (ns) } \\
$(\mathrm{ns})$ & $(\mathrm{ns})$ & $\Delta V=-20 \mathrm{~V}$ & $\Delta V=+16 \mathrm{~V}$ \\
\cline { 3 - 4 } 2 & 40 & 7.8 & 2.3 \\
5 & 40 & 7.6 & 2.3 \\
10 & 41 & 7.6 & 2.4 \\
20 & 44 & 7.5 & 2.4 \\
50 & 64 & 7.8 & 2.6 \\
\hline
\end{tabular}

was found that the pulse shapes produced by the spectroscopy amplifiers are not the same for every shaping time setting. This implies that the factors $A_{1}-A_{3}$ in (6) are not the same for every shaping time, making fitting meaningless.

The noise component proportional to $\tau$ rises with increasing $\Delta V$, as can be seen in Fig. 7. This is due to increasing amplification of the bulk leakage current through the APD, and possibly an increasing excess noise factor (see (6) and (9)). The APD capacitance hardly changes in the voltage range considered [20], implying that the $1 / \tau$-proportional series white noise and the $\tau$-independent $1 / f$ series noise components remain nearly constant. Because of these effects, the optimum shaping shifts to shorter time constants with increasing $\Delta V$, as can be observed in Fig. 7.

The thermal noise of the CSP feedback resistor is given by the last term in (9). With $R_{f}=100 \mathrm{M} \Omega$ and $T=300 \mathrm{~K}$, we find $2 k_{b} T / R_{f}=8.3 \cdot 10^{-29} \mathrm{~A}^{2} / \mathrm{Hz}$. The surface and bulk leakage currents $I_{l s}$ and $I_{l b}$ of the same type of APD were measured elsewhere [20], yielding values of $0.4 \mathrm{nA}$ and $0.02 \mathrm{nA}$, respectively. According to the CSP manufacturer, $I_{l g}$ is much smaller than the APD leakage currents, and may be neglected. At $M=50$, and assuming that $J=1.75$ [33], (9) then yields a parallel white noise contribution due only to detector leakage currents of $1.4 \cdot 10^{-26} \mathrm{~A}^{2} / \mathrm{Hz}$. The feedback resistor thermal noise may thus be neglected.

2) Time Resolution: In our experiments, the time constants for differentiation and integration are in general unequal in the fast branch, because the differentiation time constant is fixed. Consequently, a more complex expression for $E N C_{f}$ than (6) applies, see Appendix. However, increasing $M$ increases the parallel noise in this branch also, causing the integration time constant $\tau_{i}$ that minimizes $E N C_{f}$, and also the $\tau_{i}$ that optimizes timing, to shift towards shorter time constants. In Table III, the timing resolution as a function of $\tau_{i}$ is presented at two bias voltage settings, as well as the $10 \%-90 \%$ rise time of the signals. The uncertainty on these timing measurements was approximately $0.1 \mathrm{~ns}$. Although small, the expected effect is indeed observed: at $\Delta V=-20 \mathrm{~V}$, the best timing is achieved at $\tau_{i}=20 \mathrm{~ns}$, while at $\Delta V=+16 \mathrm{~V}$, this is at $\tau_{i}=2-5 \mathrm{~ns}$.

Time resolution measurements as a function of the APD bias, up to close to the breakdown voltage $\left(\Delta V_{b} \sim 18 \mathrm{~V}\right)$ are shown Fig. 8 for crystals LYSO10-P2 (10 mm, 32 channels) and LYSO20-P2 (20 mm, 64 channels). These measurements were performed with $\tau_{i}=2 \mathrm{~ns}$ and an energy threshold of $250 \mathrm{keV}$. Due to its increased number of noise sources, the time resolution of the 64-channel detector is poorer than that of the 32-channel detector. The strong dependence on $\Delta V$ observed

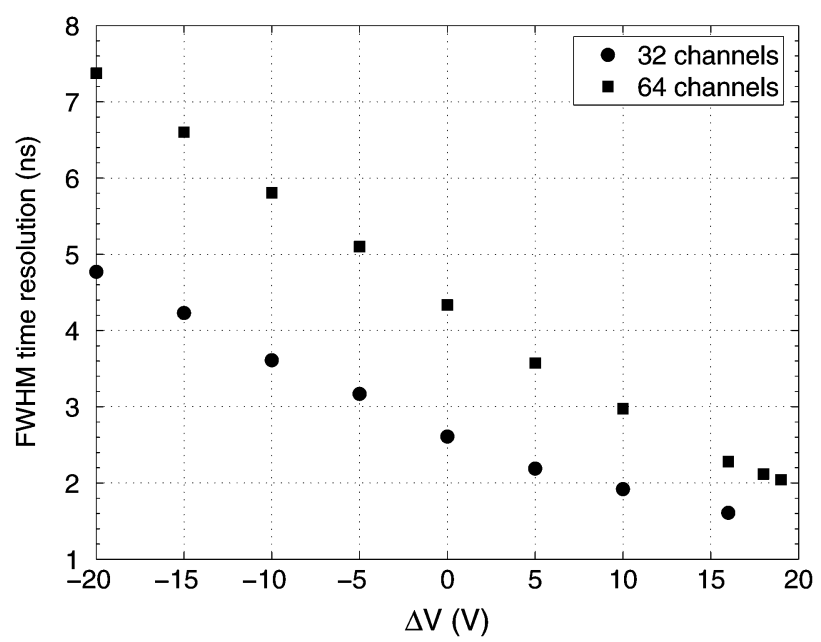

Fig. 8. Time resolution as a function of APD bias voltage, measured with crystals LYSO10-P2 (32 channels) and LYSO20-P2 (64 channels), using an TFA integration time constant of $2 \mathrm{~ns}$. The error bars fall within the markers.

in both cases may be understood by rewriting (12), splitting $E N C_{f}$ in an unamplified and an amplified part, and using the fact that $\bar{N}_{e}$ is directly proportional to $\bar{M}$ :

$$
\sigma_{A} \sim \frac{\sqrt{\beta_{1}+\beta_{2} J \bar{M}^{2}}}{\bar{M} / t_{r}} .
$$

Here, $\beta_{2}$ accounts for the component of the parallel white noise that is amplified in the APD, while $\beta_{1}$ contains all other, unamplified, noise components (see also (6) and (9)). Both $\beta_{1}$ and $\beta_{2}$ are independent of $M$, but do depend on the shaping network. Taking the derivative of (16) with respect to $\bar{M}$, treating $J$ as a constant, results in:

$$
\frac{\mathrm{d} \sigma_{A}}{\sigma_{A}}=-\frac{\beta_{1}}{\left(\beta_{1}+\beta_{2} J \bar{M}^{2}\right)} \frac{\mathrm{d} \bar{M}}{\bar{M}} .
$$

A numerical estimation presented in the appendix indicates that $\beta_{2} \ll \beta_{1}$, and so for small $M, \beta_{2} J \bar{M}^{2} \ll \beta_{1}$. In the latter regime, (17) thus reduces to $\mathrm{d} \sigma_{A} / \sigma_{A}=-\mathrm{d} \bar{M} / \bar{M}$, and a fractional increase $\mathrm{d} \bar{M} / \bar{M}$ results in a fractional decrease $\mathrm{d} \sigma_{A} / \sigma_{A}$ of the same magnitude. This explains the strong bias dependence of $\sigma_{A}$ at lower $\Delta V$. At higher $\Delta V$, the term $\beta_{2} J \bar{M}^{2}$ becomes more dominant, decreasing the dependence of $\sigma_{A}$ on $M$. At high $\Delta V$, an increase of $J$ could in principle cause a degradation of the time resolution, but this effect is not observed in the experiments.

The best time resolution, measured with the 32-channel detector at $\Delta V=+16 \mathrm{~V}$ against the $\mathrm{BaF}_{2}$-PMT-detector, was $1.6 \mathrm{~ns}$ FWHM. The corresponding time spectrum is presented in Fig. 9. The best time resolution obtained with the 64-channel detector was $2.0 \mathrm{~ns}$. The coincidence time resolution of two APD-detectors can be approximated as $\sigma_{A}^{\prime}=\sqrt{2} \sigma_{A}$, neglecting the contribution of $\sigma_{P}$. This yields $\sim 2.3 \mathrm{~ns}$ for two coincident 32-channel detectors and $\sim 2.8 \mathrm{~ns}$ for two 64-channel detectors.

3) Energy Resolution: A pulse height spectrum of crystal LYSO10-P2 at $511 \mathrm{keV}$ and $\Delta V=0 \mathrm{~V}(M \sim 50)$ is presented in Fig. 10. The corresponding FWHM energy resolution was $10.8 \%$. This value is close to the $9.7 \%$ measured with the 


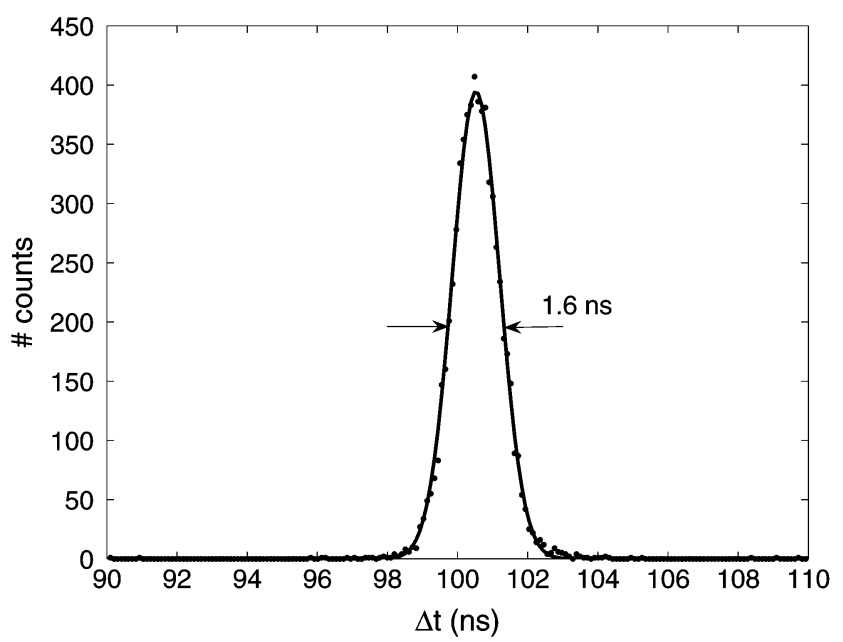

Fig. 9. Time spectrum of LYSO10-P2 at $\Delta V=+16 \mathrm{~V}$ and a TFA integration time constant of $2 \mathrm{~ns}$. The solid line represents a Gaussian fit through the data.

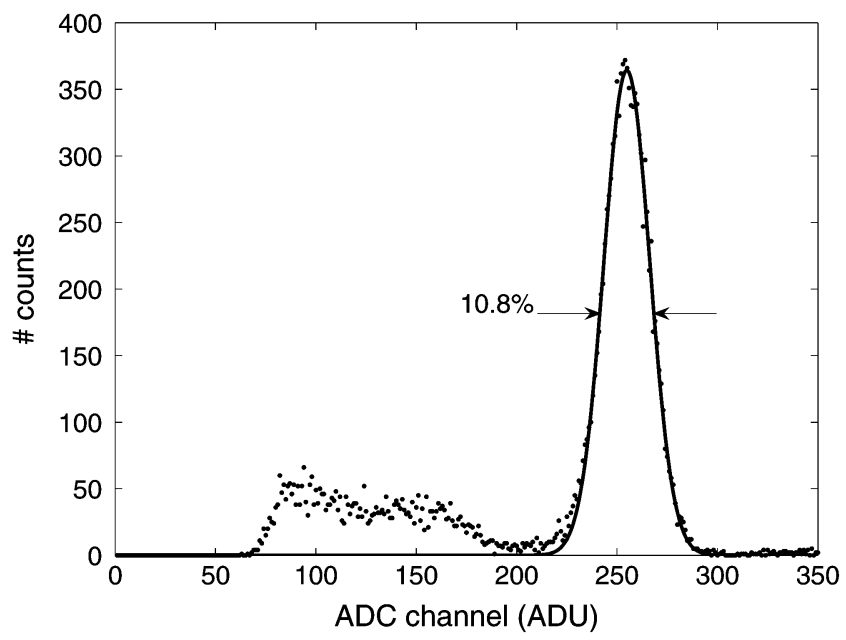

Fig. 10. Pulse height spectrum at $511 \mathrm{keV}, \Delta V=0 \mathrm{~V}$ and a shaping time of $0.2 \mu$ s of crystal LYSO10-P2. The solid line represents a Gaussian fit through the data.

LAAPD gain (see Fig. 6). Despite the considerable dead space between the pixels of the APD array of $\sim 60 \%$, the total active APD area coupled to the crystal is $81.92 \mathrm{~mm}^{2}$, while for the LAAPD this is $100 \mathrm{~mm}^{2}$. This corresponds to a difference in active area of only $18 \%$.

An overview of the energy resolutions and the different model contributions at the same bias voltage is listed in Table IV for several crystals. The term describing the excess scintillation photon variance dominates the other terms.

The energy resolutions reported here are better than those reported previously [37]. This is largely attributed to the improved quality of the crystals used in this study. Additionally, the APD arrays used were of a more recent production batch. Thus, they may have a lower excess noise factor and lower leakage current than previous batches.

A comparison between the calculated and measured energy resolutions of crystal LYSO10-P2 as a function of the APD bias is presented in Fig. 11. In contrast with the LAAPD results, it is observed that the calculated values underestimate the mea-

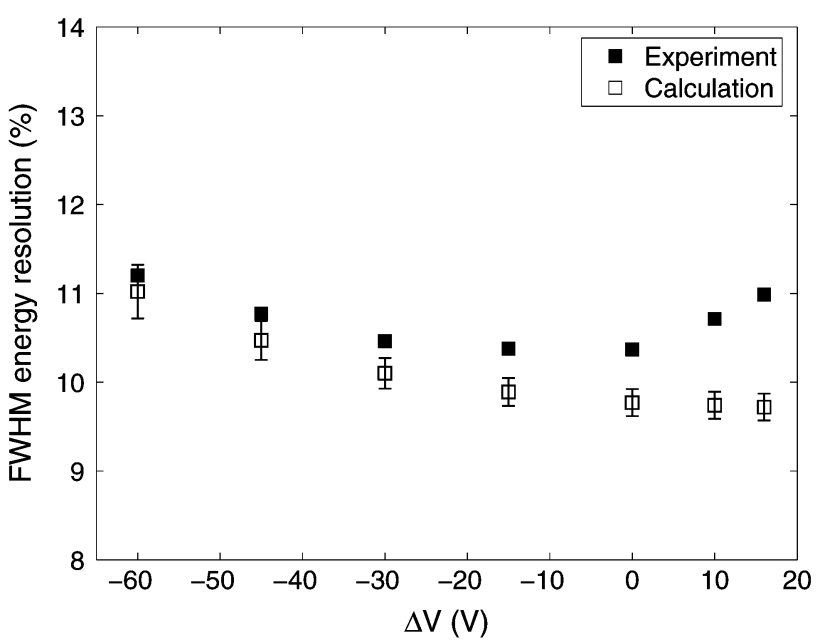

Fig. 11. Measured and calculated energy resolution of LYSO10-P2 as a function of APD bias voltage at an amplifier shaping time of $0.2 \mu \mathrm{s}$. The error bars indicate a $1 \sigma$ uncertainty. The error bars of the experimental data fall within the markers.

surements, and that the model does not describe the experimentally observed deterioration of the energy resolution at high bias. Similar results were obtained with crystal LYSO20-P2 coupled to two APD arrays. The observed differences may be caused by an increasing excess noise factor at increasing APD gain, an effect that was neglected in the model. However, a value of $J$ of $\sim 4$ would be needed to account for the difference at the highest bias if no other effects would play a role. This is a high value compared to measurements with these devices presented elsewhere [33].

Additional peak broadening may be caused by differences between the individual APD channels, which cause a dependence of the detector response on the position of interaction in the crystal. Using $5.89 \mathrm{keV} \mathrm{X}$-rays from an ${ }^{55} \mathrm{Fe}$-source, these inter-channel gain differences were found to be bias dependent, increasing from a relative standard deviation of $3.4 \%$ at $\Delta V=$ $-40 \mathrm{~V}$ to $4.9 \%$ at $\Delta V=+10 \mathrm{~V}$. The influence of these gain differences was investigated with Monte Carlo simulations in Geant4 [38], by irradiating a $20 \times 10 \times 10 \mathrm{~mm}^{3}$ Teflon-wrapped LYSO crystal coupled to one APD array with a beam of $511 \mathrm{keV}$ photons. Approximately 100 events were recorded, optically tracking the scintillation photons and scoring the number incident on each APD pixel in each event. Taking into account an excess noise factor of 1.75 and an equivalent noise charge of $750 \mathrm{e}^{-}$for each pixel, the energy resolution was determined in two situations. In the first, all APD pixels had the same gain, while in the second, the pixels were given the same relative gains as found with the ${ }^{55} \mathrm{Fe}$-measurements at $\Delta V=+10 \mathrm{~V}$. All other parameters were kept the same for this comparison. The energy resolutions found were $10.1 \pm 0.1 \%$ with equal channel gains and $10.2 \% \pm 0.1 \%$ with unequal channel gains. Thus, no significant peak broadening due to gain differences between the APD pixels was observed.

4) Spatial Resolution: Fig. 12 represents a histogram of positioning errors obtained with crystal LYSO10-P2 along the $x$-direction, containing data from all beam positions. The corresponding spatial resolution, obtained by linear interpolation of 


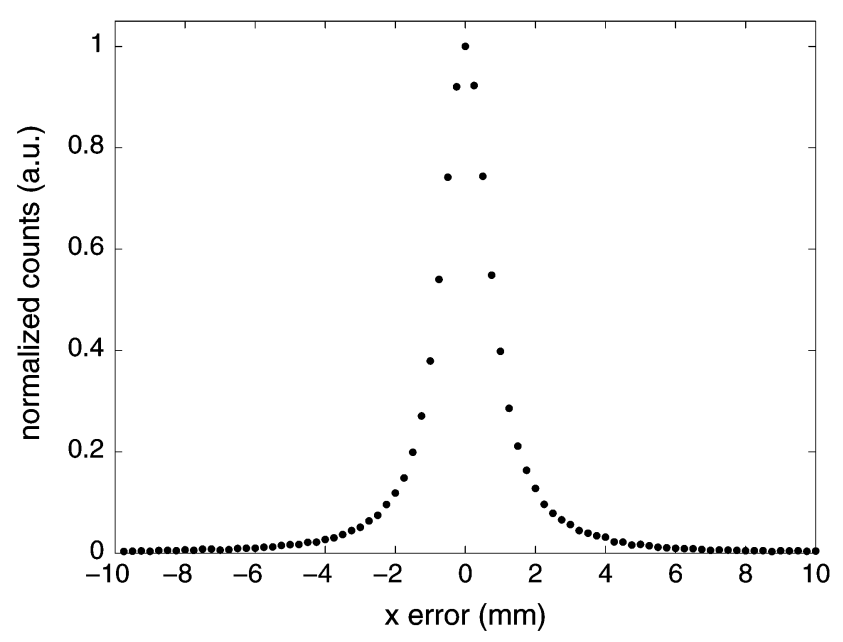

Fig. 12. Positioning error histogram obtained with crystal LYSO10-P2 along the $x$-direction, containing data from all beam positions. The corresponding spatial resolution was $1.64 \mathrm{~mm}$ FWHM and $4.42 \mathrm{~mm}$ FWTM.

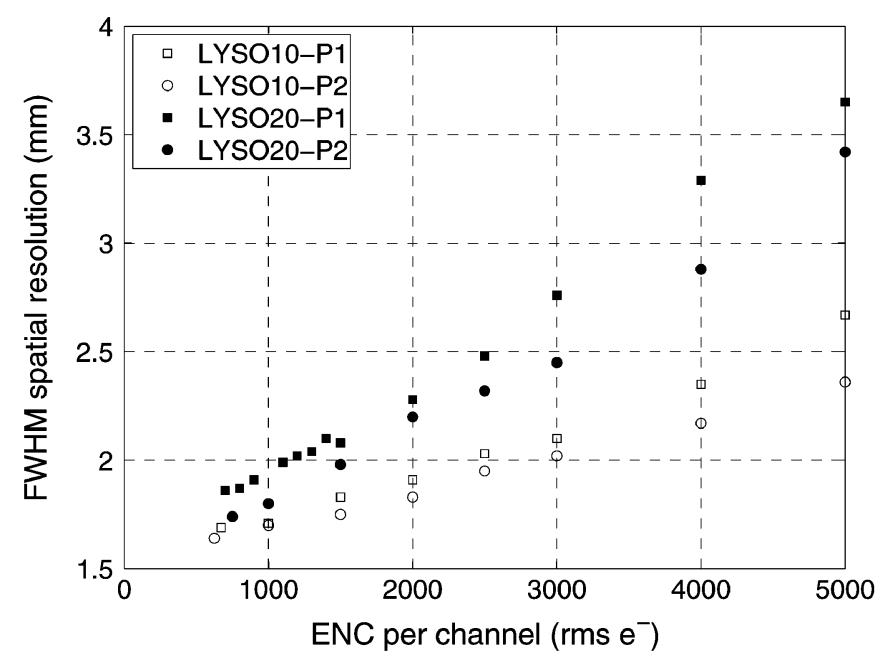

Fig. 13. Spatial resolution of several crystals as a function of the equivalent noise charge per channel, at fixed $\Delta V=0 \mathrm{~V}$. The results include the influence of the $\sim 0.9 \mathrm{~mm}$ FWHM beam. The $10-\mathrm{mm}$ (32 channel) detectors are represented by open markers, the $20-\mathrm{mm}$ ( 64 channel) detectors by solid markers.

the histogram, was $1.64 \mathrm{~mm}$ FWHM and $4.42 \mathrm{~mm}$ FWTM, not corrected for the $\sim 0.9 \mathrm{~mm}$ FWHM measurement beam.

An analysis of the influence of the e-h pair yield on the spatial resolution is presented in Table V. Both between the two 10 -mm crystals and between the two 20 -mm crystals, a slight improvement of the spatial resolution with increasing e-h pair yield is observed.

The influence of the equivalent noise charge on the spatial resolution was investigated by adding random Gaussian noise to the measured data sets by software. The amplitude of the added noise was equal for all channels. At each ENC value, a new reconstruction of the data was performed to estimate the spatial resolution.

The results of this analysis are presented in Fig. 13. The 64-channel detectors (solid markers) are clearly more affected by the noise than the 32-channel detectors (open markers), in accordance with (14). Compared to a 32-channel detector, each pixel in a 64-channel detector receives approximately half
TABLE IV

OVERVIEW OF ENERGY RESOLUTION CONTRIBUTIONS AT $\Delta V=0$

\begin{tabular}{cccccc}
\hline Crystal & $\begin{array}{c}\Delta E / E \\
(\%)\end{array}$ & $\begin{array}{c}\bar{N}_{e} \\
\left(\mathrm{e}^{-}\right)\end{array}$ & $\frac{\alpha^{2}-1}{\bar{N}_{p h}}$ & $\frac{J}{\bar{N}_{e} / \bar{M}}$ & $\frac{E N C^{2}}{\bar{N}_{e}^{2}}$ \\
\hline LYSO10-P1 & 11.0 & 370000 & $1.82 \cdot 10^{-3}$ & $2.55 \cdot 10^{-4}$ & $0.99 \cdot 10^{-4}$ \\
LYSO10-P2 & 10.8 & 411000 & $1.50 \cdot 10^{-3}$ & $2.30 \cdot 10^{-4}$ & $0.80 \cdot 10^{-4}$ \\
LYSO20-P1 & 11.9 & 304000 & $1.57 \cdot 10^{-3}$ & $3.11 \cdot 10^{-4}$ & $2.92 \cdot 10^{-4}$ \\
LYSO20-P2 & 11.0 & 374000 & $1.25 \cdot 10^{-3}$ & $2.53 \cdot 10^{-4}$ & $1.93 \cdot 10^{-4}$ \\
\hline
\end{tabular}

TABLE V

Spatial Resolution of SEVERAl LYSO CRYstals With DIFFERENT PHOTO-ELECTRON YIELDS, NOT CORRECTED FOR THE WIDTH OF THE MEASUREMENT BEAM

\begin{tabular}{|c|c|c|c|c|c|}
\hline \multirow[t]{2}{*}{ Sample } & \multirow{2}{*}{$\begin{array}{l}\bar{N}_{e} / \bar{M} \\
\left(\mathrm{e}^{-/ 511)}\right.\end{array}$} & \multicolumn{2}{|c|}{$\mathrm{x}$-resolution (mm) } & \multicolumn{2}{|c|}{ y-resolution $(\mathrm{mm})$} \\
\hline & & FWHM & FWTM & FWHM & FWTM \\
\hline LYSO10-P1 & 6858 & 1.69 & 4.57 & 1.80 & 4.79 \\
\hline LYSO10-P2 & 7606 & 1.64 & 4.42 & 1.70 & 4.33 \\
\hline LYSO20-P1 & 5631 & 1.84 & 5.09 & 1.94 & 5.56 \\
\hline LYSO20-P2 & 6928 & 1.74 & 4.76 & 1.88 & 5.03 \\
\hline
\end{tabular}

TABLE VI

SPATIAL RESOlution OF LYSO20-P2 AS A FunCTION OF THE APD BiAS VOLTAGE, NOT CORRECTED FOR THE WIDTH OF THE MEASUREMENT BEAM

\begin{tabular}{ccccccc}
\hline \multirow{2}{*}{$\Delta V$} & $E N C / N_{e}$ & \multicolumn{2}{c}{ x-resolution (mm) } & \multicolumn{2}{c}{ y-resolution (mm) } \\
$(\mathrm{V})$ & & FWHM & FWTM & & FWHM & FWTM \\
\cline { 3 - 4 } \cline { 6 - 7 }-30 & $3.2 \cdot 10^{-2}$ & 1.84 & 5.15 & & 1.98 & 5.78 \\
-15 & $2.3 \cdot 10^{-2}$ & 1.78 & 4.85 & & 1.86 & 4.99 \\
0 & $1.8 \cdot 10^{-2}$ & 1.74 & 4.76 & & 1.88 & 5.03 \\
+10 & $1.4 \cdot 10^{-2}$ & 1.78 & 5.00 & & 1.90 & 5.12 \\
+16 & $1.4 \cdot 10^{-2}$ & 1.80 & 5.17 & & 1.91 & 5.19 \\
\hline
\end{tabular}

the number of scintillation photons. Adding equal amounts of noise per channel therefore results in a poorer SNR per channel in the 64-channel case. Between the two 32-channel detectors, the crystal with the highest photon yield is least affected by the added noise (see Table V); the same is observed between the two 64-channel detectors. Furthermore, it is noted that the spatial resolution clearly deteriorates even when only little noise is added. This is an indication that electronic noise forms a non-negligible contribution to the spatial resolution, in contrast to the energy resolution. Again, this is in accordance with (14). For example, for crystal LYSO20-P2 for which $n=64$, the terms $n J /\left(\bar{N}_{e} / \bar{M}\right)$ and $n\left(E N C / \bar{N}_{e}\right)^{2}$ amount to $1.62 \cdot 10^{-2}$ and $1.24 \cdot 10^{-2}$, respectively, while the scintillation photon term remains $1.25 \cdot 10^{-3}$ (see also Table IV).

The spatial resolution as a function of the bias voltage measured with sample LYSO20-P2 is shown in Table VI. In the second column, the total electronic noise term $E N C / N_{e}$ is also indicated. Although this term is lowest at the highest bias, the optimum spatial resolution is not observed there, but around $\Delta V=0 \mathrm{~V}$. The observed deterioration at high bias may again be due to an increase of $J$, just as for the energy resolution (see Fig. 11).

All results presented here include the influence of the width of the experimental photon beam. In a PET system, this influence is not present. The beam had an estimated width of $\sim 0.9 \mathrm{~mm}$ FWHM, constituting a significant contribution to the results. However, correcting the results by deconvolution of the beam 
is not trivial, as it is divergent and has a non-Gaussian shape. A Monte-Carlo based procedure to do so will be discussed elsewhere [25]. It is estimated that with an infinitely narrow beam, the intrinsic detector spatial resolution of LYSO10-P2 at $\Delta V=$ $0 \mathrm{~V}$ is approximately $1.26 \mathrm{~mm}$ FWHM.

\section{CONCLUSION}

An analysis of the energy, timing and spatial resolutions of monolithic scintillator detectors was presented. Good agreement between a model of the energy resolution and experiments with a crystal coupled to a single pixel LAAPD was obtained. The energy resolutions observed with scintillators coupled to APD arrays were in the order of 10-11\% FWHM, but discrepancies between model calculations and experiments of up to $\sim 1.2 \%$ FWHM were found at the highest APD gains. It was shown that the energy resolution is dominated by the variance in the number of scintillation photons produced per event, while the APD excess noise factor and electronic noise contributions are of less importance. For good time and spatial resolutions, however, minimization of the electronic noise is necessary, and should thus be taken into account for example in the design of a front-end application specific integrated circuit (ASIC). Our model predicts that the excess noise factor also forms an important contribution to the spatial resolution, setting an important criterion for APDs in this application. Spatial resolutions down to $1.64 \mathrm{~mm}$ were obtained. The results were not corrected for the influence of the $\sim 0.9 \mathrm{~mm}$ FWHM beam; it is estimated that an intrinsic detector spatial resolution of $\sim 1.26 \mathrm{~mm}$ FWHM may be achieved with an infinitely narrow beam.

Coincidence timing resolutions of less than 3 ns were shown to be feasible. The best timing performance was observed operating the APD at the highest possible bias voltage. Although the best energy and spatial resolutions were achieved at lower bias, the deterioration of these two quantities at higher bias was found to be small. It is therefore likely that the best performance of a scanner based on these detectors in terms of image spatial resolution and count rate performance will be found when operating the APD arrays close to the breakdown voltage.

\section{APPENDIX \\ EQuivalent NoISE CHARGE OF THE FAST AMPLIFIER BRANCH}

In the fast branch of the CAEN N568BB amplifiers, the CSP tail pulses are differentiated with a fixed time constant $\tau_{d}$. The pulses are subsequently integrated in the TFA, with an adjustable time constant $\tau_{i}$. This system can be modeled as a CR-RC circuit, of which the equivalent noise charge in the general case of unequal time constants can be written as [18]:

$$
E N C_{f}^{2}=\frac{A}{q^{2}}\left(a C_{\mathrm{tot}}^{2}+\tau_{d} \tau_{i} b\right)
$$

where

$$
A=\frac{\left(\tau_{d}-\tau_{i}\right)^{2}}{2 \tau_{d} \tau_{i}\left(\tau_{d}+\tau_{i}\right)^{2}\left[\left(\frac{\tau_{d}}{\tau_{i}}\right)^{\frac{\tau_{i}}{\tau_{d}+\tau_{i}}}-\left(\frac{\tau_{d}}{\tau_{i}}\right)^{\frac{\tau_{d}}{\tau_{d}+\tau_{i}}}\right]^{2}} .
$$

TABLE VII

ESTIMATED NOISE PARAMETERS IN THE FAST AMPLIFIER

\begin{tabular}{|c|c|c|c|c|c|}
\hline $\begin{array}{c}a \\
\left(\mathrm{~V}^{2} / \mathrm{Hz}\right)\end{array}$ & $\begin{array}{l}C_{t o t} \\
(\mathrm{pF})\end{array}$ & $\begin{array}{c}I_{l s} \\
(\mathrm{nA})\end{array}$ & $\begin{array}{c}I_{l b} \\
(\mathrm{nA})\end{array}$ & $\beta_{1}$ & $\beta_{2}$ \\
\hline $1.1 \cdot 10^{-19}$ & 26 & 0.4 & 0.02 & $2.3 \cdot 10^{-32}$ & $1.8 \cdot 10^{-37}$ \\
\hline
\end{tabular}
BRANCH FOR A SINGLE CHANNEL

The $1 / f$ noise components are omitted in this analysis for the sake of clarity.

Expressions for the terms $\beta_{1}$ and $\beta_{2}$ introduced in the time resolution model can now be given (see (16)), considering that $\beta_{2}$ contains only the parallel white noise contribution which is due to the amplified portion of the leakage current, and $\beta_{1}$ contains all other noise sources. Using (9), it follows that

$$
\beta_{1}=\frac{A}{q^{2}}\left(a C_{\text {tot }}^{2}+\tau_{d} \tau_{i} q I_{l s}\right)
$$

and

$$
\beta_{2}=\frac{A \tau_{d} \tau_{i} I_{l b}}{q}
$$

For $\tau_{d}=100 \mathrm{~ns}$ and $\tau_{i}=2 \mathrm{~ns},(19)$ results in $A=2.9 \cdot 10^{8}$ $\mathrm{s}^{-2}$. In Table VII, estimates of the noise parameters of a single channel are listed. The parameter $a$ was obtained using (7), taking $\Gamma=0.7, T=300 \mathrm{~K}$ and $g_{m}=50 \mathrm{mS}$. The total capacitance $C_{\text {tot }}$ is the sum of $C_{\mathrm{APD}}, C_{\mathrm{FET}}, C_{f}, C_{\mathrm{tst}}$ and $C_{\text {stray }}$. The APD capacitance $C_{\mathrm{APD}}$ and the FET gate-source capacitance $C_{\mathrm{FET}}$ are both approximately $10 \mathrm{pF}$, the CSP feedback capacitance $C_{f}=1.4 \mathrm{pF}$ and the test capacitance $C_{t}=1.80 \mathrm{pF}$. Assuming a stray capacitance of $3 \mathrm{pF}$, this results in $C_{\text {tot }}=26 \mathrm{pF}$. The surface and bulk leakage currents were estimated at 0.4 and $0.02 \mathrm{nA}$, respectively [20]. The resulting values of $\beta_{1}$ and $\beta_{2}$ are also listed in Table VII. It is clear that $\beta_{2} \ll \beta_{1}$, a result that is not affected by the omission of $1 / f$ noise components from the analysis, as these would be included in $\beta_{1}$.

\section{ACKNOWLEDGMENT}

The authors would like to thank Craig Woody of Brookhaven National Laboratories for kindly supplying the printed circuit boards used to read out the APD arrays, and Rob Hollander for fruitful discussions and important suggestions.

\section{REFERENCES}

[1] D. P. McElroy, W. Pimpl, B. J. Pichler, M. Rafecas, T. Schuler, and S. I. Ziegler, "Characterization and readout of MADPET-II detector modules: Validation of a unique design concept for high resolution small animal PET,' IEEE Trans. Nucl. Sci., vol. 52, no. 1, pp. 199-204, Jan. 2005.

[2] K. Ziemons et al., "The ClearPET (TM) project: Development of a 2nd generation high-performance small animal PET scanner," Nucl. Instrum. Meth. Phys. Res. A, vol. 537, no. 1-2, pp. 307-311, Jan. 2005.

[3] T. Tsuda et al., "A four-layer depth of interaction detector block for small animal PET," IEEE Trans. Nucl. Sci., vol. 51, no. 5, pp. 2537-2542, Oct. 2004.

[4] J. Seidel, J. J. Vaquero, and M. V. Green, "Resolution uniformity and sensitivity of the NIH ATLAS small animal PET scanner: Comparison to simulated LSO scanners without depth-of-interaction capability," IEEE Trans. Nucl. Sci., vol. 50, no. 5, pp. 1347-1351, Oct. 2003.

[5] S. Surti, J. S. Karp, A. E. Perkins, R. Freifelder, and G. Mühllehner, "Design evaluation of A-PET: A high sensitivity animal PET camera," IEEE Trans. Nucl. Sci., vol. 50, no. 5, pp. 1357-1363, Oct. 2003. 
[6] Y. C. Tai et al., "MicroPET II: Design, development and initial performance of an improved microPET scanner for small-animal imaging," Phys. Med. Biol., vol. 49, pp. 1519-1539, 2003.

[7] J. A. Correia, C. A. Burnham, D. Kaufman, and A. J. Fischman, "Development of a small animal PET imaging device with resolution approaching $1 \mathrm{~mm}$," IEEE Trans. Nucl. Sci., vol. 46, no. 3, pp. 631-635, Jun. 1999.

[8] S. Weber et al., "Recent results of the TierPET scanner," in Proc. IEEE Nuclear Science Symp. Medical Imaging Conf. Rec., Seattle, WA, vol. 3, pp. 1603-1607.

[9] A. Del Guerra, G. Di Domenico, M. Scandola, and G. Zavatti, "High spatial resolution small animal YAP-PET," Nucl. Instrum. Meth. Phys. Rese. A, vol. 409, pp. 537-541, 1998.

[10] P. M. Bloomfield, R. Myers, S. P. Hume, T. J. Spinks, A. A. Lammertsma, and T. Jones, "Three-dimensional performance of a small-diameter positron emission tomograph," Phys. Med. Biol., vol. 42, pp. 389-400, 1997.

[11] R. Lecomte et al., "Initial results from the Sherbrooke avalanche photodiode positron tomograph," IEEE Trans. Nucl. Sci., vol. 43, no. 3, pp. 1952-1957, Jun. 1996.

[12] D. Clément, R. Frei, J. Loude, and C. Morel, "Development of a 3D position sensitive scintillation detector using neural networks," in Proc. IEEE Nucl. Sci. Symp. Med. Imag. Conf. Rec., 1998, vol. 3, pp. $1448-1452$.

[13] P. Bruyndonckx, S. M. A. Léonard, J. Liu, S. P. K. Tavernier, P. Szupryczynski, and A. Fedorov, "Study of spatial resolution and depth of interaction of APD-based PET detector modules using light sharing schemes," IEEE Trans. Nucl. Sci., vol. 50, no. 5, pp. 1415-1419, Oct. 2003.

[14] M. C. Maas, D. J. van der Laan, D. R. Schaart, P. Bruyndonckx, C. Lemaître, and C. W. E. van Eijk, "Performance of APD-based monolithic-crystal detectors for small animal PET," in Proc. IEEE Nucl. Sci. Symp. Conf. Rec., 2005, vol. 4, pp. 2017-2021.

[15] J. Birks, The Theory and Practice of Scintillation Counting. New York: Pergamon, 1964.

[16] Geant4 User's Guide for Application Developers, Geant4 7.1 june 2005 ed., [Online]. Available: http://wwwasd.web.cern.ch/wwwasd/ geant4/G4UsersDocuments/UsersGuides/ForApplicationDeveloper/html71/index.html

[17] D. J. van der Laan, M. C. Maas, D. R. Schaart, P. Bruyndonckx, S. Léonard, and C. W. E. van Eijk, "Using Cramér-Rao theory combined with Monte Carlo simulations for the optimization of monolithic scintillator PET detectors," IEEE Trans. Nucl. Sci., vol. 53, no. 3, pp. 1063-1070, Jun. 2006.

[18] E. Gatti, P. F. Manfredi, M. Sampietro, and V. Speziali, "Suboptimal filtering of 1/f-noise in detector charge measurements," Nucl. Instrum. Meth. Phys. Res. A, vol. 297, no. 3, pp. 467-478, 1990.

[19] G. Bertuccio and A. Pullia, "A method for the determination of the noise parameters in preamplifying systems for semiconductor radiation detectors," Revi. Sci. Instrum., vol. 64, no. 11, pp. 3294-3298, 1993.

[20] J. B. Mosset, S. Saladino, J. F. Loude, and C. Morel, "Characterisation of arrays of avalanche photodiodes for small animal positron emission tomography," Nucl. Instrum. Meth. Phys. Res. A, vol. 504, no. 1-3, pp. 325-330, 2003.

[21] M. Moszynski and B. Bengtson, "Status of timing with plastic scintillation detectors," Nucl. Instrum. Meth., vol. 158, pp. 1-31, 1979.
[22] C. Fiorini, A. Gola, A. Longoni, F. Perotti, and L. Struder, "Timing properties of silicon drift detectors for scintillation detection," IEEE Trans. Nucl. Sci., vol. 51, no. 3, pp. 1091-1097, Jun. 2004.

[23] D. J. van der Laan, D. R. Schaart, M. C. Maas, P. Bruyndonckx, and C. W. E. van Eijk, "Spatial resolution in position-sensitive scintillation detectors: statistics and noise," to be published.

[24] M. C. Maas et al., "Experimental characterization of novel small animal PET detector modules based on scintillation crystal blocks read out by APD arrays," in Proc. IEEE Nucl. Sci. Symp. Conf. Rec., 2004, vol. 5, pp. 2942-2946.

[25] M. C. Maas, D. J. van der Laan, C. W. E. van Eijk, P. Bruyndonckx, C. Lemaître, and D. R. Schaart, "Model analysis of the line spread function of monolithic scintillator detectors for high resolution PET," to be published.

[26] J. Boisvert, J. Montroy, L. Jostad, B. Zhou, and M. Szawlowski, "Improved large-area avalanche photodiodes for scintillation detection in calorimetry," in Proc. Nucl. Sci. Symp. Conf. Rec., 1996, vol. 1, pp. 16-20, vol. 1.

[27] M. Goyot, B. Ille, P. Lebrun, and J. P. Martin, "Performances of a preamplifier silicon photodiode readout system associated with large BGO crystal scintillators," Nucl. Instrum. Meth. Phys. Res. A, vol. 263, no. 1, pp. 180-187, Jan. 1988.

[28] M. Kapusta, P. Crespo, D. Wolski, M. Moszynski, and W. Enghardt, "Hamamatsu S8550 APD arrays for high-resolution scintillator matrices readout," Nucl. Instrum. Meth. Phys. Res. A, vol. 504, no. 1-3, pp. 139-142, 2003.

[29] P. Dorenbos, J. T. M. de Haas, and C. W. E. van Eijk, "Non-proportionality in the scintillation response and the energy resolution obtainable with scintillation crystals," IEEE Trans. Nucl. Sci., vol. 42, no. 6, pp. 2190-2202, Dec. 1995.

[30] B. Carriço, "Quality control of APDs," in LIP. Lisbon, Portugal: , 2006, private communication.

[31] J. P. Pansart, "Avalanche photodiodes for particle detection," Nucl. Instrum. Meth. Phys. Res. A, vol. 387, no. 1-2, pp. 186-193, 1997.

[32] M. Moszynski, M. Szawlowski, M. Kapusta, and M. Balcerzyk, "Large area avalanche photodiodes in scintillation and X-rays detection," Nucl. Instrum. Meth. Phys. Res. A, vol. 485, no. 3, pp. 504-521, 2002.

[33] J.-B. Mosset, "Développement d'un Module de Détection Phoswich LSO/LuYAP Pour le Prototype de Caméra à Positrons ClearPET," Ph.D. dissertation, Ecole Polytechnique Fédérale de Lausanne, Lausanne, Switzerland, 2006.

[34] A. Ochi, Y. Nishi, and T. Tanimori, "Study of a large area avalanche photodiode as a fast photon and a soft X-ray detector," Nucl. Instrum. Meth. Phys. Res. A, vol. 378, no. 1-2, pp. 267-274, 1996.

[35] T. M. Cover and P. E. Hart, "Nearest neighbor pattern classification," IEEE Trans. Inf. Theory, vol. IT-13, no. 1, p. 21, Jan., 1967.

[36] M. C. Maas et al., "Experimental characterization of monolithic-crystal small animal PET detectors read out by APD arrays," IEEE Trans. Nucl. Sci., vol. 53, no. 3, pp. 1071-1077, Jun. 2006.

[37] P. Bruyndonckx et al., "Neural network-based position estimators for PET detectors using monolithic LSO blocks," IEEE Trans. Nucl. Sci., vol. 51, no. 5, pp. 2520-2525, Oct. 2004.

[38] S. Agostinelli et al., "GEANT4-A simulation toolkit," Nucl. Instrum. Meth. Phys. Res. A, vol. 506, no. 3, pp. 250-303, 2003. 